\title{
Multi-period Portfolio Selection Using the Finite Difference Method
}

\author{
Xing $\mathrm{Yu}^{*}$ \\ Department of Mathematics \& Applied Mathematics, Hunan University of humanities, science and technology, Loudi, \\ 417000, P.R. China
}

\begin{abstract}
We investigate a continuous-time mean-variance portfolio selection problem. Different from the general stochastic dynamic programming approach, such as using Hamilton-Jacobi-Bellman (HJB) equation, this paper adopts the Lagrange duality method and the finite difference approach to derive explicit closed-form expressions for the efficient investment strategy and the mean-variance efficient frontier.
\end{abstract}

Keywords: Continuous time mean-variance, finite difference method, optimization, portfolio selection.

\section{INTRODUCTION}

The effective way to allocation capital among various assets is portfolio selection. In 1952, Markowitz proposed the famous mean-variance model for single-period portfolio selection, which set up a fundamental basis for modern finance [1]. There is a deficiency in Markowitz's model. As we know, due to the changes on the risk and return preferences, the limitation of the budget of an investor, the portfolio has obvious dynamic characteristics. From this angle, many scholars extend the single period portfolio to the multi period ones. Yong [2] etc proposed a multi-stage mean-variance portfolio selection model by using interval analysis. Chen [3] illustrated that it is difficult to solve the multi-stage portfolio selection problems. Duan Li and Wang-Lung Ng [4] derived the analysis solution for the multi-stage mean-variance model. Charnes [5] is the first to extent the single period portfolio selection to the multi-stage case with the dynamic programming method, but he did not get the analysis solution. Dantzig and Infanger [6] solved the multi-stage portfolio selection with stochastic programming method. Due to the complexity of stochastic programming method, Ben-Tal [7] adopted robust substitution method to deal with the problem. In contrast to the mainstream stochastic programming approach to multi-period optimization, Giuseppe Carlo Calafiore [8] proposed solution approach was based on a specific affine parameterization of the recourse policy, which allows us to obtain a sub-optimal but exact and explicit problem formulation in terms of a convex quadratic program. Yao [9] used the Lagrange duality method and the dynamic programming approach, and derived explicit closed-form expressions for the efficient investment strategy and the meanvariance efficient frontier. El-Ghatwary [10] proposed a new fault detection (FD) approach for non-linear stochastic

*Address correspondence to this author at the Department of Mathematics \& Applied Mathematics, Hunan University of humanities, science and technology, Loudi, 417000, P.R. China; E-mail: hnyuxing@ 163.com systems. Optimization algorithm based on minimizing the residual covariance matrix is used to obtain a robust FD for global system behavior.

The existing literatures have adopted stochastic programming method to solve the model. However, to the best of our best knowledge, there is no study on the numerical method. It is well known that the finite difference method is one of the main numerical methods for solving partial differential equations. Because the data computer can store only limited data and co operations, so any kind of suitable for computer solving method, must take the continuous time discretization, which finally turns into a limited form of equation. This paper proposes the optimal portfolio model, using the Lagrange duality method and the finite difference approach. We derive explicit closed-form expressions for the efficient investment strategy and the mean-variance efficient frontier. The advantage of this algorithm lies in the last moment from the portfolio effective period to push down to the start time, so as to achieve the intended purpose.

The rest of this paper is organized as follows. We establish in Section 2 the continuous-time mean-variance model. In Section 3, we introduce the principle of the finite difference method. we construct and solve the model's Lagrangian problem in Section 4. We derive explicitly the efficient investment strategy and the efficient frontier for the original mean-variance model In Section 5.We conclude our paper in Section 6.

\section{THE MODEL}

Suppose there is a market in which $n+1$ assets are traded continuously. One of the assets is the risk free asset whose price process is calculated as:

$$
\left\{\begin{array}{c}
d P_{0}(t)=r(t) P_{0}(t) d t \\
P_{0}(0)=p_{0}>0
\end{array}, t \in[0, T]\right.
$$


where $r(t)>0$ is the risk free interest rate. The other $n$ risk assets such as stocks whose price processes $P_{1}(t), P_{2}(t) \cdots P_{n}(t)$ satisfy the following stochastic differential equation:

$$
\left\{\begin{array}{c}
d P_{i}(t)=P_{i}(t)\left\{b_{i}(t) d t+\sum_{j=1}^{n} \sigma_{i j}(t) d W_{j}(t)\right\}, t \in[0, T] \\
P_{i}(0)=p_{i}>0
\end{array}\right.
$$

where $b_{i}(t)>0$ is the appreciation rate, and $\sigma_{i}(t)=\left(\sigma_{i 1}(t), \sigma_{i 2}(t) \cdots \sigma_{i n}(t)\right)$ is the volatility of the stocks. The covariance matrix is defined as

$\sigma(t)=\left(\begin{array}{l}\sigma_{1}(t) \\ \sigma_{2}(t) \\ \mathrm{L} \\ \sigma_{n}(t)\end{array}\right)=\left(\sigma_{i j}(t)\right)_{n \times n}$

For simplicity, we introduce the following notations

$w_{i}$ the proportion invested in the ith assets

$1-\sum_{i=1}^{n} w_{i}$ the proportion invested in the 0 th or the risk free asset

The investor's wealth $x(t)$ satisfies the following stochastic differential equation

$$
\begin{aligned}
& d x(t)=x(t)\left\{\begin{array}{l}
{\left[b_{0}(t)+\sum_{i=1}^{n} w_{i}(t)\left(b_{i}(t)-b_{0}(t)\right)\right] d t+} \\
{\left[\sigma_{0}(t)+\sum_{i=1}^{n} w_{i}(t)\left(\sigma_{i}(t)-\sigma_{0}(t)\right)\right] d W(t)}
\end{array}\right\} \\
& x(0)=x_{0}
\end{aligned}
$$

Regard the parameters $\mu, \sigma^{2}$ are constant firstly, let

$$
\begin{aligned}
& \mu=b_{0}(t)+\sum_{i=1}^{n} w_{i}(t)\left(b_{i}(t)-b_{0}(t)\right) \\
& \sigma^{2}=\sigma_{0}(t)+\sum_{i=1}^{n} w_{i}(t)\left(\sigma_{i}(t)-\sigma_{0}(t)\right)
\end{aligned}
$$

then

$$
d x(t)=x(t)\left\{\mu d t+\sigma^{2} d W(t)\right\}
$$

\section{THE OPTIMAL CONTINUOUS-TIME MEAN- VARIANCE PORTFOLIO SELECTION MODEL}

The aim of the continuous-time mean-variance portfolio selection model is to find the optimal strategy minimizing the variance while attaining a given level of the expectation.
The continuous-time mean-variance portfolio selection model is formed as the following stochastic optimal problem

$$
\begin{aligned}
& \min _{w(\cdot)} \operatorname{Var}[x(T)]=E[x(T)-u]^{2}(\mathrm{M} 1) \\
& \text { s.t } E[X(T)]=u
\end{aligned}
$$

where $u$ is the terminal expectation an investor requires to achieve, which is a constant. In our paper, it is supposed that there is no transaction cost for simple.

Introducing the Lagrange method to transform (M1) to the following stochastic optimal control problem

$$
\min E[X(T)-u]^{2}+2 \lambda[E X(T)-u](\mathrm{M} 2)
$$

Let $a=\lambda-u$, solving (M2) equal to solve the following optimal problem

$$
\min E[X(T)+a]^{2}(\mathrm{M} 3)
$$

We consider now the portfolio selection problem for a truncated problem beginning at an arbitrary time $\mathrm{t}$ with wealth $x$

$$
V(x, t)=E\left((X(T)+a)^{2} \mid x_{t}=x\right)
$$

The optimal problem (M3) means to choose $w_{i}(\cdot)$ satisfy $\min V(x, t)$, that is to solve

$$
\min _{w_{i}(\cdot)} V(x, t)(\mathrm{M} 4)
$$

According to Black-Scholes formulation, $V(x, t)$ satisfy the following equation

$$
V_{t}+\mu x V_{x}+\frac{1}{2} \sigma^{2} x^{2} V_{x x}-r V=0
$$

And the boundary conditions

$$
V(x, T)=(x+a)^{2}
$$

\section{FINITE DIFFERENCE METHODS}

It aims to find the expression of $V(x, t)$. The existing literatures almost all adopt stochastic dynamic programming using HJB equation. Innovatively, this paper uses finite difference method to solve the problem. Some scholars use four order differential structure calculation scheme, whose accuracy is not necessarily high, we adopt two order differential structure for simple.

Constructing grid

$$
\begin{aligned}
& Q=\{(i \Delta x, j \Delta t) \mid i=1,2 \cdots M, j=1,2 \cdots N\} \\
& \Delta \mathrm{t}=\frac{T}{N}, N \Delta t=T
\end{aligned}
$$


At every grid points, define $V_{i, j}=V(i \Delta x, j \Delta t)$, by finite difference method

$V_{t}=\frac{V_{i, j+1}-V_{i, j}}{\Delta t}, V_{x}=\frac{V_{i+1, j}-V_{i, j}}{\Delta x}$,

$V_{x x}=\frac{V_{i+1, j}-2 V_{i, \mathrm{j}}+V_{i-1, j}}{\Delta x^{2}}$

According to (2.5), we have

$\frac{V_{i, j+1}-V_{i, j}}{\Delta t}+\mu i\left(V_{i+1, j}-V_{i, j}\right)+\frac{1}{2} \sigma^{2}\left(V_{i+1, j}-2 V_{i, j}+V_{i-1, j}\right)-r V_{i, j}=0$

We can find

$\left(\frac{1}{\Delta t}+i \mu+r-\sigma^{2}\right) V_{i, \mathrm{j}}=\left(\frac{1}{2} \sigma^{2}+i \mu\right) V_{i+1, \mathrm{j}}+\frac{1}{\Delta t} V_{i, \mathrm{j}+1}+\frac{1}{2} \sigma^{2} V_{i-1, \mathrm{j}}$

It means that, if we obtain any three points value, the fourth point's value could be deduced by (2.8). When $t=T$, the value of $V_{T, j}$ at the grid points respectively are $(j \Delta x+a)^{2}$.

From the operation rules, it can obtain all the node values, especially node values at the $X$ axis whose vertical axis $x=0, \Delta x, 2 \Delta x \cdots M \Delta x$. That is, each column node value relatively known. The optimal problem is aimed to choose the minimal value of each column. The steps of the algorithm,

Calculate the node value of the last column, from bottom to top respectively are

$a^{2},(\Delta x+a)^{2},(2 \Delta x+a)^{2} \cdots(M \Delta x+a)^{2}$.

According to the equation (2.8), it can be obtained all the node values.

Choose $w(j \Delta t)$ to get the minimal value of each column node values.

$$
w^{*}(j \Delta t)=\frac{\left(\sigma(j \Delta t) \sigma^{\prime}(j \Delta t)\right)^{-1} V_{x} B(j \Delta t)}{V_{x x} x}
$$

where $V_{x}, V_{x x}$ follow to (2.7).

\section{EMPIRICAL ANALYSES AND COMPARATIVE STUDY}

\subsection{Empirical Analyses}

In this part, it focuses on an empirical research of an optimal portfolio model with five risk assets in Chinese market which show a good momentum in 2013 . We choose day data of five stocks NO 603126, 300389, 300390, 603009 and 300391 from 2013-01-04 09:01:00 to 2014-6-4 15:00:00, 58320 data in total, which are from CSMAR database in China. From previous data, the expectation vector and covariance matrix are directly calculated:

$$
\begin{aligned}
& \mu(t)=(0.4264,0.3112,0.2620,0.1127,0.0824) \\
& \sigma(t) \sigma^{\prime}(t)=\left(\begin{array}{lllll}
0.1911 & 0.1087 & 0.1732 & 0.1098 & 0.2173 \\
0.1087 & 0.1346 & 0.0922 & 0.1179 & 0.1868 \\
0.1732 & 0.0922 & 0.2572 & 0.2383 & 0.1364 \\
0.1098 & 0.1179 & 0.2383 & 0.1663 & 0.1887 \\
0.2173 & 0.1868 & 0.1364 & 0.1887 & 0.3352
\end{array}\right)
\end{aligned}
$$

If the initial wealth is 1 and investment horizon is $T=1$, risk-free rate $r=0.05$ solving the model, we obtain the optimal investment strategy

$w^{*}\left(x_{i}\right)=\frac{0.5371 u-0.0589 e^{0.6173}}{x_{i}}\left(\begin{array}{l}0.2078 \\ 1.2813 \\ -0.5924 \\ 0.2735 \\ -0.3026\end{array}\right)$

where $x_{i}$ is the current wealth value, and $u$ is the given level of expected terminal wealth. And the efficient frontier of the optimal portfolio model is given by

$\operatorname{Var}\left(x_{T}\right)=\frac{e^{-0.6173}}{1-e^{-0.6173}}\left(E\left(x_{T}\right)-e^{-0.05}\right)^{2}$

\subsection{Comparative Study}

In order to contrast study for proposed the finite difference method, the results compared between the general stochastic dynamic programming approach and our approach. It shows that the efficient boundary curves almost coincide. And westling claims that the results are robust under different structure parameters.

\section{CONCLUSION}

Different from the general stochastic dynamic programming approach to solve multi-period portfolio selection problem, we adopt the finite difference approach instead. Numerical example shows that the algorithm is feasible and effective. And we derive explicit closed-form expressions for the efficient investment strategy and the mean-variance efficient frontier. This paper can be extended in several directions. For example, it may be necessary to include constraints of transaction costs, which may have significant effect on the optimal solution of the problem.

\section{CONFLICT OF INTEREST}

The author confirms that this article content has no conflict of interest.

\section{ACKNOWLEDGEMENTS}

This research is supported by scientific research fund of Hunan provincial education department (NO 2A077). 


\section{REFERENCES}

[1] H.M. Markowitz, "Portfolio selection", Journal of Finance, vol. 7, pp. 77-91, 1952.

[2] Y.J. Liu, G.Z. Wei, and Z. Pu,"A multi-period portfolio selection optimization model bu using interval analysis", Economic Modelling, vol. 33, pp. 113-119, 2013.

[3] P.Chootinan, and A.Chen, "Constraint handling in genetical gorithms using agradient- based repair method", Computers \& Operations Research, vol. 33, pp. 2263-2281, 2006.

[4] D. Li, and N. Wan-Lung, "Optimal dynamic portfolio selection: multi period mean-variance formulation", Mathematical Finance, vol. 10, pp. 387-406, 2000.

[5] A. Charnes, and W.W. Cooper, Management models and the industrial applications of linear programming, Wiley: New York, 1968.
G.B. Dantzig, and G. Infanger, "Multi-stage stochastic linear programs for portfolio optimization", Annals of Operations Research, vol. 45, pp. 59-76, 1993.

[7] A. Ben-Tal and A. Nemirovski, "Robust solution of uncertain linear program", Operations Research Letters, vol. 25, pp. 1-13, 1999.

[8] G.C. Calafiore,"Multi-period portfolio optimization with linear control policies", Automatica, vol. 44, pp. 2463-2473, 2008.

[9] H. Yao, Z. Li, and S. Chen, "Continuous-time mean-variance portfolio selection with only risky assets", Economic Modelling, vol. 364, pp. 244-251, 2014.

[10] M.G. El-ghatwary and S.X. Ging, "Robust fuzzy fault detection for non-linear stochastic dynamic systems", Open Automation and Control Systems Journal, vol. 2, pp. 45-53, 2009.

Received: December 12, 2014

(C) Xing Yu; Licensee Bentham Open.

This is an open access article licensed under the terms of the Creative Commons Attribution Non-Commercial License (http://creativecommons.org/licenses/by-nc/3.0/) which permits unrestricted, non-commercial use, distribution and reproduction in any medium, provided the work is properly cited. 